\title{
Endometrioid adenocarcinoma with choriocarcinomatous differentiation: A case report and review of the literature
}

\author{
MITSUAKI ISHIDA and HIDETOSHI OKABE \\ Department of Clinical Laboratory Medicine and Division of Diagnostic Pathology, \\ Shiga University of Medical Science, Otsu, Shiga 520-2192, Japan
}

Received January 21, 2013; Accepted June 13, 2013

DOI: $10.3892 / \mathrm{ol} .2013 .1431$

\begin{abstract}
A choriocarcinomatous component is rarely present in carcinomas of certain sites and few cases of choriocarcinomatous differentiation in endometrioid adenocarcinoma have been reported. The present study reports a case of endometrioid adenocarcinoma of the uterine corpus with choriocarcinomatous differentiation, and discusses the clinicopathological features of this rare tumor. A 59-year-old post-menopausal female presented with abnormal vaginal bleeding. Magnetic resonance imaging demonstrated a relatively well-circumscribed tumor in the uterine corpus and a total cystectomy was subsequently performed. A histopathological examination revealed two distinct components in the uterine corpus tumor. The first component comprised $\sim 80 \%$ of the tumor and was composed of poorly-differentiated endometrioid adenocarcinoma. The remaining component consisted of mononucleated and syncytial giant cells containing rich eosinophilic cytoplasm and large pleomorphic nuclei with coarse chromatin. An immunohistochemical analysis revealed that these syncytial giant cells were positive for $\beta$-human chorionic gonadotropin (hCG). Therefore, a diagnosis of endometrioid adenocarcinoma with choriocarcinomatous differentiation was confirmed. The clinicopathological features of nine previously reported cases of this tumor were analyzed in addition to the present case. The majority of the patients were post-menopausal. Endometrial choriocarcinoma may be considered to have a highly aggressive clinical course, since nine of the 10 cases displayed metastases and four patients succumbed to the disease. The pathogenesis of the choriocarcinomatous component is not well understood. However, genetic studies have demonstrated that conventional carcinoma and choriocarcinomatous components share common genetic alterations. The choriocarcinomatous
\end{abstract}

Correspondence to: Dr Mitsuaki Ishida, Department of Clinical Laboratory Medicine and Division of Diagnostic Pathology, Shiga University of Medical Science, Tsukinowa-cho, Seta, Otsu, Shiga 520-2192, Japan

E-mail: mitsuaki@belle.shiga-med.ac.jp

Key words: endometrioid adenocarcinoma, choriocarcinomatous differentiation, chorionic gonadotrophin component represents aberrant differentiation of the conventional carcinoma, however, genetic analyses of endometrioid adenocarcinoma with choriocarcinomatous differentiation have not been performed.

\section{Introduction}

Choriocarcinoma is a rare malignant neoplasm composed of mononucleated and multinucleated trophoblasts, mainly arising in the uterus of pregnant females and in the ovaries. A choriocarcinomatous component is rarely present in carcinomas of certain sites, including the lung (1), breast (2), esophagus (3), stomach $(4,5)$, colon $(6,7)$ and urinary system $(8)$. Furthermore, uterine corpus carcinomas with choriocarcinomatous differentiation have rarely been reported (9-17). The present study describes a case of endometrioid adenocarcinoma of the uterine corpus with choriocarcinomatous differentiation and discusses the clinicopathological features of this rare tumor. Written informed consent was obtained from the patient.

\section{Case report}

Patient. A 59-year-old post-menopausal female with scleroderma and diabetes mellitus presented with abnormal vaginal bleeding. A physical examination revealed the presence of pyometra and magnetic resonance imaging demonstrated a relatively well-circumscribed tumor, measuring $30 \mathrm{~mm}$ in diameter, in the fundus of the uterus (Fig. 1). Swelling of the internal and external iliac and paraaortic lymph nodes was also observed. A clinical diagnosis of a malignant uterine corpus tumor was suspected, and a total cystectomy and bilateral salpingo-oophorectomy were performed, with dissection of the pelvic and paraaortic lymph nodes. Serum $\beta$-human chorionic gonadotropin (hCG) levels were not measured.

Materials and methods. Formalin-fixed, paraffin-embedded tissue blocks were cut into $3-\mu \mathrm{m}$ thick sections, then deparaffinized and rehydrated. Each section was stained with hematoxylin and eosin and used for immunostaining. Immunohistochemical analyses were performed using an autostainer (Benchmark XT system; Ventana Medical System, Tucson, AZ, USA) according to the manufacturer's instructions. The following primary antibodies were used: Mouse monoclonal antibody against CA125 (Ov185:1; Novocastra 
Table I. Clinicopathological features of endometrioid adenocarcinoma with choriocarcinomatous differentiation.

\begin{tabular}{|c|c|c|c|c|c|c|}
\hline Case no. & Age (years) & $\begin{array}{l}\text { Histopathology of } \\
\text { coexisting tumor }\end{array}$ & Metastases or invasion & $\begin{array}{l}\text { Histopathology at } \\
\text { metastatic sites }\end{array}$ & $\begin{array}{l}\text { Outcome, } \\
\text { months }\end{array}$ & Reference \\
\hline 1 & 70 & WD & Brain, lung, liver, kidneys & Choriocarcinoma & DOD, 14 & 9 \\
\hline 2 & 78 & PD & Pelvic lymph nodes & NA & DOD, 1.5 & 10 \\
\hline 3 & 48 & PD & Lungs & NA & AWD, 2 & 10 \\
\hline 4 & 63 & Adenocarcinoma & Lungs, liver, peritoneum & Same as primary site & DOD, 14 & 10 \\
\hline 5 & 83 & MD & Lungs & NA & AWD, 1 & 11 \\
\hline 6 & 68 & $\begin{array}{l}\text { PD, clear cell and } \\
\text { serous papillary }\end{array}$ & Pelvic lymph nodes & $\begin{array}{l}\text { Serous papillary } \\
\text { adenocarcinoma }\end{array}$ & NED, 16 & 12 \\
\hline 7 & 54 & MD & Retroperitoneum & Adenocarcinoma & DOD, 24 & 13 \\
\hline 8 & 58 & WD & Vaginal cuff & Choriocarcinoma & NED, 36 & 14 \\
\hline 9 & 42 & MD & None & & NED, 6 & 15 \\
\hline Present case & 59 & PD & Iliac and paraaortic lymph nodes & Same as primary site & NED, 2 & \\
\hline
\end{tabular}

WD, well-differentiated adenocarcinoma; PD, poorly-differentiated adenocarcinoma; MD, moderately-differentiated adenocarcinoma; NA, not available; AWD, alive with disease; DOD, died of disease; NED, no evidence of disease.

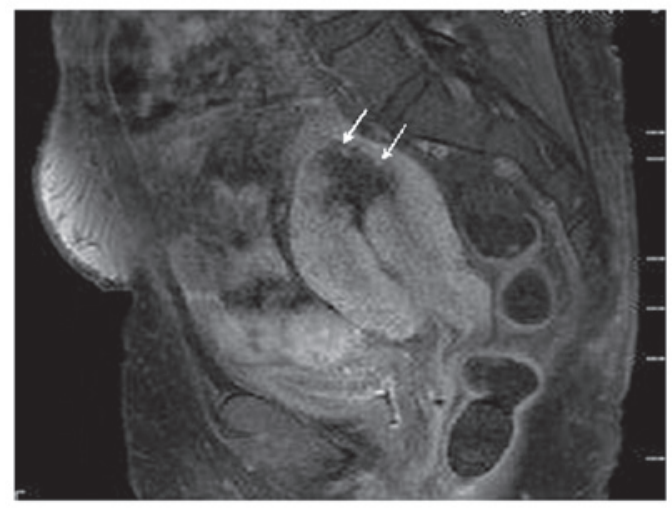

Figure 1. Magnetic resonance imaging showing a relatively well-circumscribed tumor in the fundus of the uterine corpus (arrows).

Laboratories, Ltd., Newcastle upon Tyne, UK), mouse monoclonal antibody against cytokeratin (AE1/AE3; DAKO Cytomation, Glostrup, Denmark) and rabbit polyclonal antibody against human $\beta$-hCG (Novocastra).

Histopathological findings. The uterine corpus tumor consisted of two distinct histopathological components. The first component comprised $\sim 80 \%$ of the tumor and was composed of a poorly-differentiated adenocarcinoma with extensive hemorrhage and necrosis. This area involved a proliferation of sheets or variable-sized nests of atypical epithelial cells containing large oval nuclei with coarse chromatin and small nucleoli (Fig 2A and B). These tumor cells contained a relatively rich, marginally eosinophilic cytoplasm, but no intracytoplasmic mucin was observed (Fig. 2B). Mitotic figures were frequently observed (34/10 high-power fields). Focal glandular differentiation showing cribriform glands with central necrosis was present (Fig. 2C), however, no squamous differentiation was noted. Accordingly, this component was considered to be an endometrioid adenocarcinoma. The remaining component consisted of mononucleated and syncytial-like giant cells, with a rich eosinophilic cyto- plasm and large pleomorphic nuclei with coarse chromatin (Fig. 2D). There was a transition between the endometrioid adenocarcinoma and choriocarcinomatous components (Fig. 2D). A number of lymphatic and vascular invasions were noted (Fig. 2E). The tumor had invaded deeply into the entire layer of the uterine corpus wall and serosal invasion was also noted. However, no parametrial or vaginal invasion was observed. The internal and external iliac and paraaortic lymph nodes exhibited metastatic carcinomas accompanying each of the components.

Immunohistochemical findings. Cytokeratin (AE1/AE3) was expressed in the endometrioid adenocarcinoma and choriocarcinomatous components. CA125 was expressed in the endometrioid adenocarcinoma component, but not in the choriocarcinomatous component. $\beta$-hCG was expressed in the choriocarcinomatous component, particularly in the syncytial giant cells (Fig. 3), but not in the endometrial carcinoma component. Metastatic lesions of the lymph nodes showed similar immunohistochemical features to the primary tumor and $\beta$-hCG-positive syncytial giant cells were scattered amongst the metastatic lesions.

According to the histopathological and immunohistochemical features, an ultimate diagnosis of endometrioid adenocarcinoma with choriocarcinomatous differentiation was made [pIIIC2; International Federation of Gynecology and Obstetrics (FIGO)].

\section{Discussion}

The present study describes a case of endometrioid adenocarcinoma of the uterine corpus with choriocarcinomatous differentiation. Civantos and Rywlin first reported a case of uterine corpus carcinoma (serous papillary adenocarcinoma) with choriocarcinomatous differentiation in 1972 (16). Subsequently, Savage et al reported the first case of endometrioid adenocarcinoma of the uterine corpus with choriocarcinomatous differentiation in 1987 (9). Since then, 

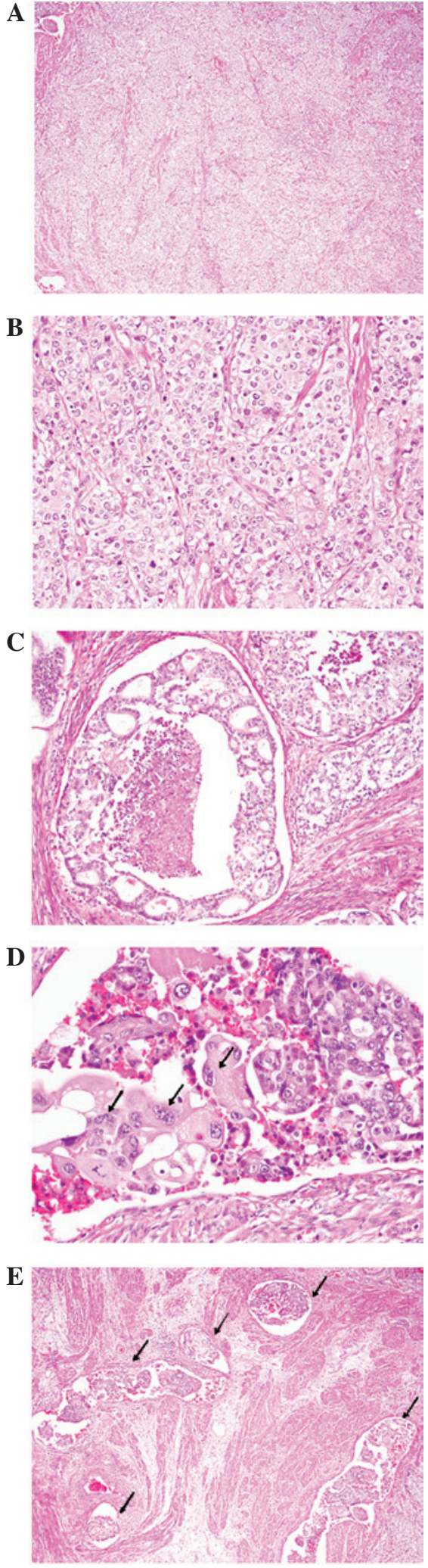

Figure 2. Histopathological findings of the uterine corpus tumor (A) Poorly-differentiated endometrioid adenocarcinoma component Proliferation of sheets or variable-sized nests of atypical epithelial cells (hematoxylin and eosin; magnification, x40). (B) Atypical epithelial cells contain a relatively rich, marginally eosinophilic cytoplasm and large nuclei with coarse chromatin and small nucleoli (hematoxylin and eosin; magnification, x200). (C) Glandular differentiation showing a cribriform structure with central necrosis (hematoxylin and eosin; magnification x100). (D) Choriocarcinomatous component. Syncytial giant cells are scattered (arrows; hematoxylin and eosin; magnification, x200). (E) Vascular and lymphatic invasions are prominent (arrows; hematoxylin and eosin; magnification, $\mathrm{x} 40$ ).

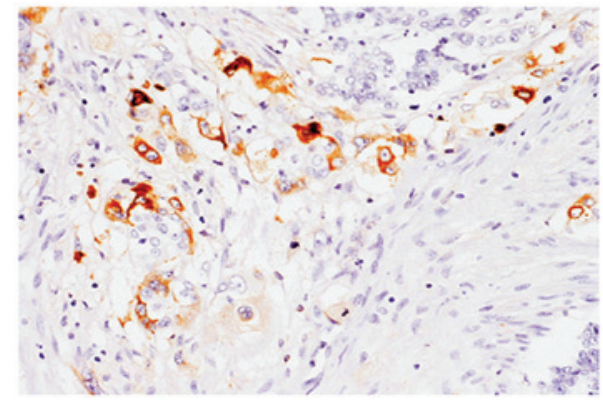

Figure 3. Immunohistochemical features of the uterine corpus tumor $\beta$-human chorionic gonadotrophin (hGC) is expressed in the syncytial giant cells (x200).

few uterine corpus adenocarcinomas with choriocarcinomatous differentiation have been reported (10-15). The most common histopathological subtype of the carcinomatous component is endometrioid adenocarcinoma (9-15), as seen in the present case. Serous papillary adenocarcinoma $(16,17)$, clear cell adenocarcinoma (18) and carcinosarcoma with choriocarcinomatous differentiation $(19,20)$ have also been documented. In addition, uterine cervical adenocarcinoma with choriocarcinomatous differentiation has also been reported (21).

Table I summarizes the clinicopathological features of nine previously reported cases of endometrioid adenocarcinoma of the uterine corpus with choriocarcinomatous differentiation, in addition to the present case. The median age of the patients was 62.3 years (range, $42-83$ years) and the majority were post-menopausal females, with the exception of the case reported by Akbulut $e t a l$, in which the patient was a 42-year-old premenopausal female (15). Abnormal vaginal bleeding and abdominal pain were the main presenting symptoms $(14,15)$. Endometrial choriocarcinoma may be considered to have a highly aggressive clinical course, since nine of the 10 cases studied showed metastases, with the common metastatic sites being the lung, liver and lymph nodes, while four patients succumbed to the disease (Table I). The histopathological features of the metastatic sites were variable; two cases, including the present case, shared the same features as the primary site (adenocarcinoma with choriocarcinomatous components) and two cases only exhibited the choriocarcinoma component (Table I).

Although the pathogenesis of the choriocarcinomatous component in non-gestational tumors is not well understood, studies suggest that the choriocarcinomatous component probably represents heterotopic or aberrant differentiation of the conventional carcinoma components, rather than the malignant transformation of germ cells $(7,22)$. Zetll et al reported a case of urothelial carcinoma with a choriocarcinomatous component and analyzed the comparative genomic hybridization of the two components (22). The study clearly demonstrated that the components shared losses of chromosomes 9 and 17p, which were characteristic genetic alterations of urothelial carcinoma, and that the choriocarcinomatous components acquired additional chromosomal losses and gains, mostly associated with poorly-differentiated urothelial carcinoma (22). The results suggest a close genetic association between urothelial carcinoma and the choriocarcinomatous component. Furthermore, 
Verbeek et al reported a case of rectal adenocarcinoma with choriocarcinomatous components and identified genetic changes that are characteristic of colorectal adenocarcinoma (losses of chromosomes $8 p$ and $18 q$ and gains of $5 p$ and 20q) in the two components, providing evidence for a common origin (7). According to these results, the two histological components of endometrioid adenocarcinoma with choriocarcinomatous differentiation may share a common genetic origin, however, genetic analyses of this rare tumor have not been performed.

In conclusion, the present study describes the 10th documented case of endometrioid adenocarcinoma of the uterine corpus with choriocarcinomatous differentiation. The clinicopathological analyses revealed that this rare tumor has a highly aggressive clinical course, with a high incidence of metastases and a high mortality rate. Therefore, identifying the choriocarcinomatous component in endometrioid adenocarcinoma is essential for establishing an adequate therapeutic strategy.

\section{References}

1. Serno J, Zeppernick F, Jäkel J, et al: Primary pulmonary choriocarcinoma: case report and review of the literature. Gynecol Obstet Invest 74: 171-176, 2012.

2. Mohammadi A and Rosa M: Carcinoma of the breast with choriocarcinomatous features. Arch Pathol Lab Med 135: 1097-1100, 2011.

3. Merimsky O, Jossiphov J, Asna N, Shmueli E, Stabsky A and Inbar M: Choriocarcinoma arising in a squamous cell carcinoma of the esophagus. Am J Clin Oncol 23: 203-206, 2000.

4. Satake N, Chikakiyo M, Yagi T, Suzuki Y and Hirose T: Gastric cancer with choriocarcinoma and yolk sac tumor components: case report. Pathol Int 61: 156-160, 2011.

5. Yoon JH, Kim MS, Kook EH, et al: Primary gastric choriocarcinoma: two case reports and review of the literatures. Cancer Res Treat 40: 145-150, 2008.

6. Harada M, Inoue $\mathrm{T}$ and Hamano $\mathrm{K}$ : Choriocarcinoma of the sigmoid colon: report of a case. Surg Today 42: 93-96, 2012.

7. Verbeek W, Schulten HJ, Sperling M, et al: Rectal adenocarcinoma with choriocarcinomatous differentiation: clinical and genetic aspects. Hum Pathol 35: 1427-1430, 2004.

8. Minamino K, Adachi Y, Okamura A, et al: Autopsy case of primary choriocarcinoma of the urinary bladder. Pathol Int 55: 216-222, 2005.
9. Savage J, Subby W and Okagaki T: Adenocarcinoma of the endometrium with trophoblastic differentiation and metastases as choriocarcinoma: a case report. Gynecol Oncol 26: 257-262, 1987.

10. Pesce C, Merino MJ, Chambers JT and Nogales F: Endometrial carcinoma with trophoblastic differentiation. An aggressive form of uterine cancer. Cancer 68: 1799-1802, 1991.

11. Kalir T, Seijo L, Deligdisch L and Cohen C: Endometrial adenocarcinoma with choriocarcinomatous differentiation in an elderly virginal woman. Int J Gynecol Pathol 14: 266-269, 1995.

12. Bradley CS, Benjamin I, Wheeler JE and Rubin SC: Endometrial adenocarcinoma with trophoblastic differentiation. Gynecol Oncol 69: 74-77, 1998.

13. Tunç M, Simşek T, Trak B and Uner M: Endometrium adenocarcinoma with choriocarcinomatous differentiation: a case report. Eur J Gynaecol Oncol 19: 489-491, 1998.

14. Yamada T, Mori H, Kanemura M, Ohmichi M and Shibayama Y: Endometrial carcinoma with choriocarcinomatous differentiation: a case report and review of the literature. Gynecol Oncol 113: 291-294, 2009.

15. Akbulut M, Tosun H, Soysal ME and Oztekin O: Endometrioid carcinoma of the endometrium with choriocarcinomatous differentiation: a case report and review of the literature. Arch Gynecol Obstet 278: 79-84, 2008.

16. Civantos F and Rywlin AM: Carcinomas with trophoblastic differentiation and secretion of chorionic gonadotrophins. Cancer 29: 789-798, 1972.

17. Horn LC, Hänel C, Bartholdt E and Dietel J: Serous carcinoma of the endometrium with choriocarcinomatous differentiation: a case report and review of the literature indicate the existence of 2 prognostically relevant tumor types. Int J Gynecol Pathol 25: 247-251, 2006.

18. Black K, Sykes P and Ostör AG: Trophoblastic differentiation in an endometrial carcinoma. Aus NZ J Obstet Gynaecol 38: 472-473, 1998

19. Khuu HM, Crisco CP, Kilgore L, Rodgers WH and Conner MG: Carcinosarcoma of the uterus associated with a nongestational choriocarcinoma. South Med J 93: 226-228, 2000.

20. Nguyen CP, Levi AW, Montz FJ and Bristow RE: Coexistent choriocarcinoma and malignant mixed mesodermal tumor of the uterus. Gynecol Oncol 79: 499-503, 2000.

21. Shintaku M, Kariya M, Shime H and Ishikura H: Adenocarcinoma of the uterine cervix with choriocarcinomatous and hepatoid differentiation: report of a case. Int J Gynecol Pathol 19: 174-178, 2000.

22. Zettl A, Konrad MA, Polzin S, et al: Urothelial carcinoma of the renal pelvis with choriocarcinomatous features: genetic evidence of clonal evolution. Hum Pathol 33: 1234-1237, 2002. 